\title{
Article \\ Application of the MiL and HiL Simulation Techniques in Stewart Platform Control Development
}

\author{
Dominik Walica *(D) and Petr Noskievič (D) \\ Department of Control Systems and Instrumentation, VSB-Technical University of Ostrava, \\ 70800 Ostrava-Poruba, Czech Republic; petr.noskievic@vsb.cz \\ * Correspondence: dominik.walica@vsb.cz
}

\begin{abstract}
During the integration phase of a system development, we are often concerned as to whether the designed control algorithm could be performed on the selected controller in real-time. One of the tools to test and validate the control scheme is the Hardware-in-the-Loop (HiL) simulation technique, which is a part of a model-based design methodology. This approach requires a simulation model of a controlled system running in a real-time loop with an intended controller and a control algorithm, which are objects of interest in this method. To perform the test, the control algorithm must be deployed to the controller such as a PLC. This paper presents a use case of the HiL technique in the design of the Stewart platform control, where the controller is PLCnext from Phoenix Contact. The control algorithm was first verified in the Model-in-the-Loop simulation (MiL) and then generated as a code from the Matlab/Simulink environment and deployed to the PLCnext, which resulted in a smoother transition from the design to the integration and testing phase. The presented method is also applicable to other controllers that support code generation.
\end{abstract}

Keywords: Hardware-in-the-Loop; model-based design; Model-in-the-Loop; programmable logic controller; Stewart platform

Citation: Walica, D.; Noskievič, P. Application of the MiL and HiL Simulation Techniques in Stewart Platform Control Development. Appl. Sci. 2022, 12, 2323. https://doi.org/ 10.3390/app12052323

Academic Editor: Dariusz Kania

Received: 30 December 2021

Accepted: 21 February 2022

Published: 23 February 2022

Publisher's Note: MDPI stays neutral with regard to jurisdictional claims in published maps and institutional affiliations.

Copyright: (C) 2022 by the authors. Licensee MDPI, Basel, Switzerland. This article is an open access article distributed under the terms and conditions of the Creative Commons Attribution (CC BY) license (https:// creativecommons.org/licenses/by/ $4.0 /)$.

\section{Introduction}

The V-Cycle (Figure 1) as defined in the VDI 2206 guideline is a systematic approach for the development of a mechatronic system [1]. The V-Cycle or V-Model is represented by a seamlessly following development stages and gradual verification and validation. The initial stage is a precise definition of the system requirements. The next step involves an analysis of the requirements and development of the general design of the system, with the support of system engineering tools and languages such as SysML or System Composer $[2,3]$. The general solution concept of the system is then split into specific domains of mechanical, electrical, and software engineering to be further concretized and then integrated to form an overall system. At this point, the interaction between the domains needs to be investigated. The design process is continuously verified and validated. The result of this cycle is a verified and validated product. The entire development cycle is also supported with modelling and simulation tools and follows the model-based design methodology [4-6].

If the development cycle has been strongly supported with modelling and simulation tools, after the domain-specific design, we have designed a simulation model of the mechatronic system and its control strategy. In case we decide to proceed with this design to the system integration phase, we need to check whether the designed control algorithm could be performed on a desired hardware in real-time $[7,8]$. Since testing the control algorithm on the real prototype can be dangerous in many scenarios, it is efficient to use Model-in-the-Loop (MiL), Software-in-the-Loop (SiL), Processor-in-the-Loop (PiL) or Hardware-in-the-Loop (HiL) techniques. MiL is often used in the early phase of development to verify the design [9], and the rest of the techniques are used later in the system integration phase $[10,11]$. 


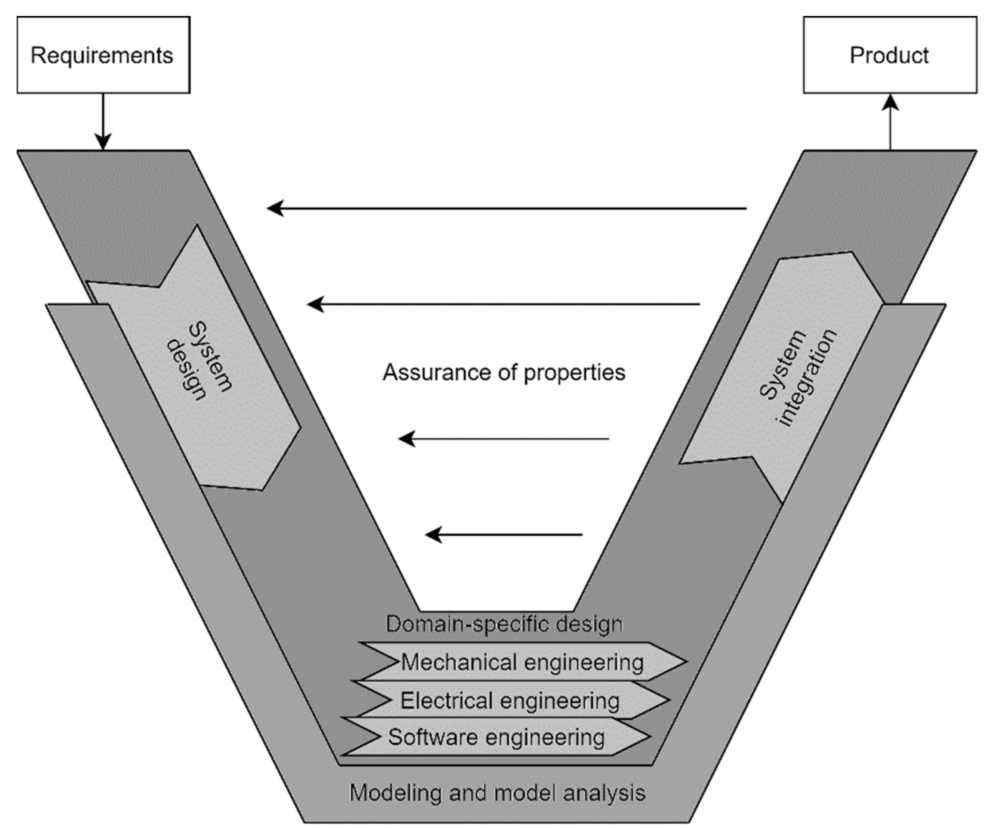

Figure 1. V-Cycle defined in VDI 2206.

MiL is performed in the simulation environment (i.e., Matlab/Simulink) where both the controller and the plant are mathematical models. SiL is performed in the simulation environment as well, but the controller is implemented as a code (i.e., $\mathrm{C} / \mathrm{C}++$ ) that is going to be implemented in the controller. PiL performs a simulation where the controller code is implemented on a development prototyping board and is connected through the I/O interface with the computer that simulates the plant. The last technique to be mentioned is HiL (Figure 2), which works on a similar principle to PiL, with the difference that the controller is run in real-time on the intended hardware and the plant simulation model runs on the real-time target. The HiL tests can also include safety functions.

Controller workstation
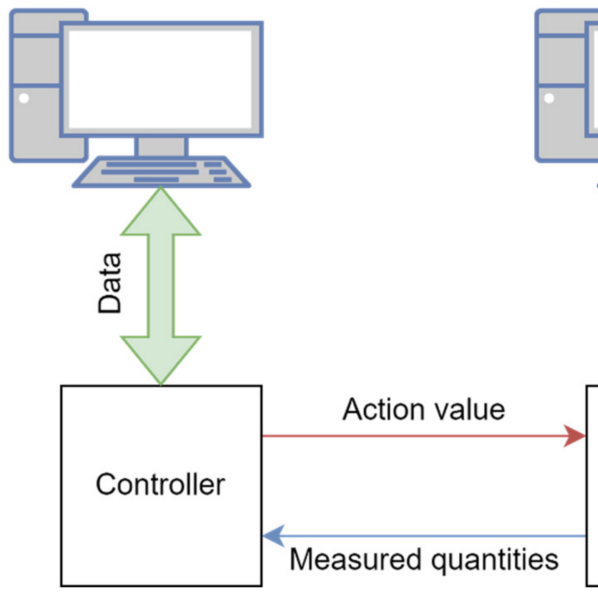

Real-time target workstation

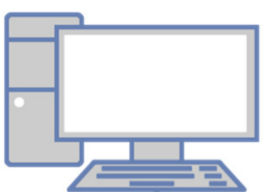

$\stackrel{\pi}{\pi}$

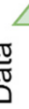

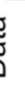

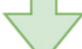

Real-time target

Figure 2. Hardware-in-the-Loop general scheme.

One of the most significant advantages of the model-based design approach is the automatic code generation [12,13]. Therefore, we had to choose a controller that is powerful enough to perform our code in real-time and supports the conversion of our controller model from the Matlab/Simulink environment, to the form that is compatible with the selected controller. For this purpose, we have chosen the PLCnext controller, which meets the defined requirements [14]. The programming code can also be created in classical PLC 
languages according to the IEC 61131-3 or in C\#, C/C++, etc. The code created by both approaches can be combined and merged into the complete control system. PLCnext can also run an HMI application for visualisation and control. Since PLCnext can run as a web server, the HMI application can be accessed through the standard web browser.

In this article, we are concerned with the transition from the design phase to the system integration phase and verification of the designed control strategy for the Stewart platform. In the first part, we describe the Stewart platform parallel mechanism. Then we present the multibody simulation model of the Stewart platform, the designed feedback control loop and the results of the MiL simulation. In the next part, we present the application of the HiL simulation technique, where the PLCnext is tested as the desired controller of the plant. The results of the HiL simulation are then compared with those of the MiL simulation. The last part also discusses the actual and potential benefits of this approach as well as the risks and obstacles that are bound to this development methodology in relation to our case.

\section{Stewart Platform}

The Stewart platform (Figure 3) is a parallel manipulator with six degrees of freedom [15]. Its advantages are mainly its positioning precision, stiffness, and high powerto-density ratio. Its disadvantage is its relatively small workspace [16-18]. Application of the Stewart platform can range from vibration isolation, motion simulators, to stabilization of a load, and more $[19,20]$. The Stewart platform consists of a base plate, six extensible legs, six upper and lower joint pairs, and a moving plate. The base plate is attached to the ground. The upper and lower joint pairs are formed by spherical and universal joints. The position and orientation of the moving plate depend on the extension of each extensible leg, which is represented by a linear actuator, which consists of a linear actuator body and a piston rod. The resulting trajectory of the moving plate is thus given by a synchronized motion of the actuators. The calculation of the required piston rod extension is done with the use of inverse kinematics [21,22].

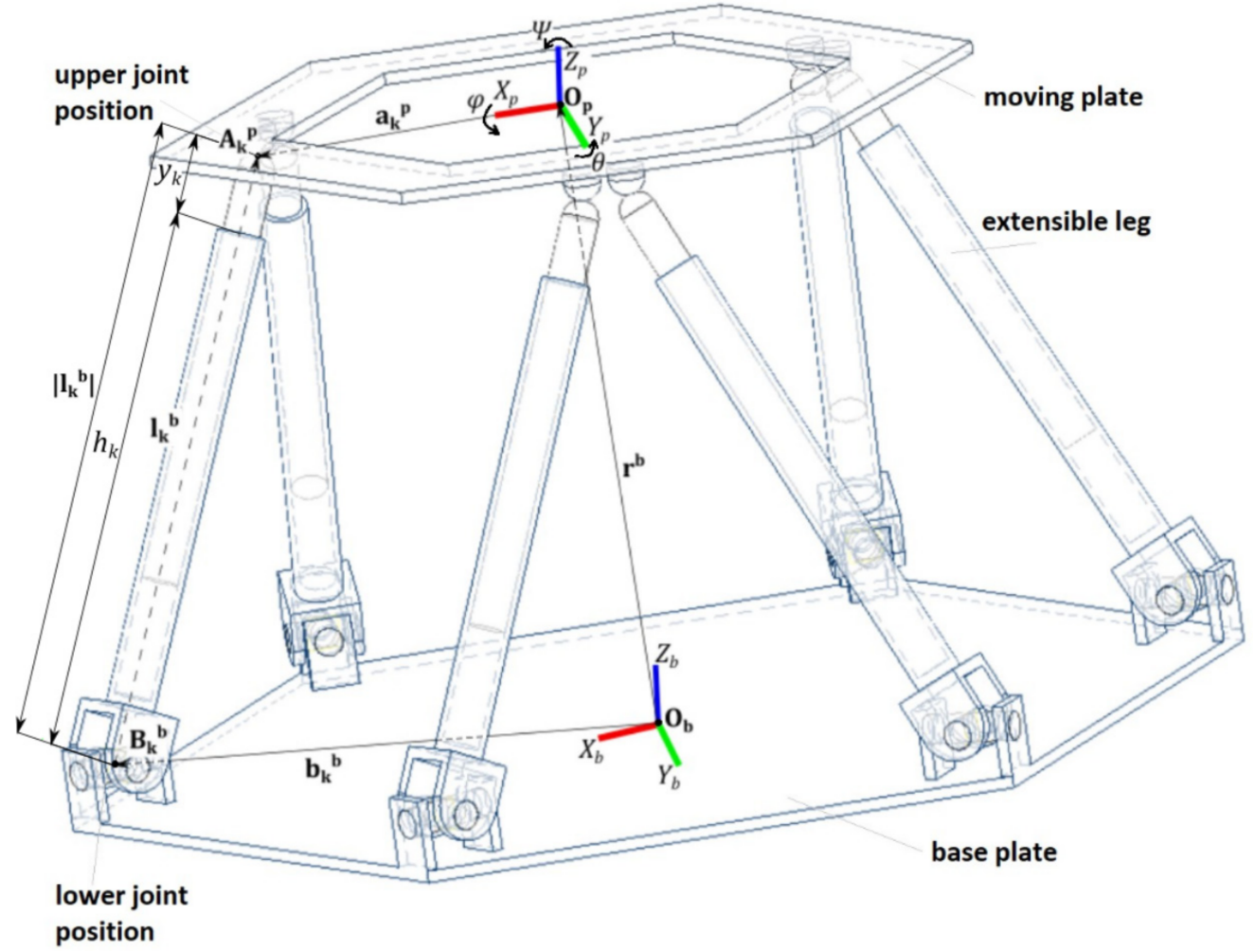

Figure 3. Schematic view of the general Stewart platform. 
As shown in Figure 3, there is a base coordinate system $\mathbf{O}_{\mathbf{b}} x_{b} y_{b} z_{b}$ fixed to the centre of the base plate. The lower joint position is given by $\mathbf{B}_{\mathbf{k}} \mathbf{b}$. The second coordinate system $\mathbf{O}_{\mathbf{p}} x_{p} y_{p} z_{p}$ is fixed to the moving plate. The upper joint position is given by $\mathbf{A}_{\mathbf{k}} \mathbf{p}$. The linear actuator body and the piston rod are related by a cylindrical joint. The linear actuator body is attached by the universal joint to the base plate. The piston rod is attached by the spherical joint to the moving plate. The position of the moving plate is given by (1). The orientation of the moving plate is given by roll $\varphi$, pitch $\theta$ and yaw $\Psi$ angles of the moving plate coordinate system with respect to the base coordinate system. The position and orientation of the moving plate is thus given by (2).

$$
\begin{gathered}
\overline{\mathbf{O}_{\mathbf{b}} \mathbf{O}_{\mathbf{p}}}=\mathbf{r}^{\mathbf{b}}=\left[x_{b}, y_{b}, z_{b}\right]^{T} \\
\mathbf{q}=\left[x_{b}, y_{b}, z_{b}, \varphi, \theta, \Psi\right]^{T}
\end{gathered}
$$

To calculate the leg length required to reach the desired $\mathbf{q}$, we need to use inverse kinematics (3). In this article, we will differentiate between desired position and orientation of the moving plate $\mathbf{q}_{\mathbf{w}}$ and an actual position and orientation of the moving plate $\mathbf{q}_{\mathbf{y}}$. Based on the structure of the Stewart platform given by the vectors of upper and lower joint position $\mathbf{a}_{\mathbf{k}} \mathbf{p}$ and $\mathbf{b}_{\mathbf{k}} \mathbf{b}$, and the desired position and orientation, we can calculate the leg vector $\mathbf{l}_{\mathbf{k}} \mathbf{b}$. $\mathbf{R}$ is a rotation matrix. $C$ and $S$ represent $\cos$ and $\sin$ functions. The leg length is then obtained from (5). The leg length consists of $h_{k}$, which is a fixed length of the linear actuator body and $y_{k}$, which represents the piston rod extension. The piston rod extension $y_{k}$ is controlled by the Stewart platform control system. The piston rod extension is given by a distance of the piston rod reference frame with respect to the linear actuator body reference frame as is described in Section 3. Note that these calculations do not consider limitations such as minimum and maximum length of the extensible legs, as well as minimum and maximum joint angles, collisions of the bodies and singularity configurations. All these factors determine the workspace of the Stewart platform and should be checked on [23].

$$
\begin{gathered}
\overline{\mathbf{B}_{\mathbf{k}}^{\mathbf{b}} \mathbf{A}_{\mathbf{k}}^{\mathbf{b}}}=\mathbf{l}_{\mathbf{k}}^{\mathbf{b}}=\mathbf{r}^{\mathbf{b}}+\mathbf{R} \mathbf{a}_{\mathbf{k}}^{\mathbf{p}}-\mathbf{b}_{\mathbf{k}}^{\mathbf{b}} \\
k=1,2, \ldots, 6 \\
\mathbf{R}=\left[\begin{array}{ccc}
C_{\Psi} C_{\theta} & C_{\Psi} S_{\theta} S_{\varphi}-S_{\Psi} C_{\varphi} & S_{\Psi} S_{\varphi}+C_{\Psi} S_{\theta} C_{\varphi} \\
S_{\Psi} C_{\theta} & C_{\Psi} C_{\varphi}+S_{\Psi} S_{\theta} S_{\varphi} & S_{\Psi} S_{\theta} C_{\varphi}-C_{\Psi} S_{\varphi} \\
-S_{\theta} & C_{\theta} S_{\varphi} & C_{\theta} C_{\varphi}
\end{array}\right] \\
\left|\mathbf{l}_{\mathbf{k}}^{\mathbf{b}}\right|=\sqrt{\mathbf{l}_{\mathbf{k}}^{\mathbf{b}} \mathbf{l}_{\mathbf{k}}^{\mathbf{b}}}=h_{k}+y_{k}
\end{gathered}
$$

\section{Model-in-the-Loop (MiL) Simulation of the Stewart Platform}

Since the development of the Stewart platform is done according to the V-Cycle, it has been supported with modelling and simulation tools, and therefore the multibody simulation model of the Stewart platform has been created. There are several methods available to build multibody simulation models of the Stewart platform.

The first one is to develop multibody simulation model with Newton-Euler, Lagrange or virtual work principle approach [24-26]; these approaches are demanding, and it is hard to implement such models in the HiL simulation. Another approach is to develop the multibody simulation model with the use of software such as MSC Adams [27], Matlab/Simulink [28], etc.

In this paper, the presented simulation model has been developed with Matlab/Simulink and a Simscape Multibody toolbox. The mentioned software allows us to build a multibody simulation model in the Simulink environment with the blocks representing reference frames, bodies, joints, etc. However, it also supports the workflow, where the multibody assembly is exported from CAD software and is imported into the Matlab/Simulink 
environment $[28,29]$. The following Stewart platform simulation model has been created with the latter mentioned approach.

In Figure 4 is the 3D visualisation of the created multibody simulation model of the Stewart platform. Its structure is similar to one presented in Figure 3, but it has specified physical properties now. The function of the extensible leg is performed by a linear actuator, and the upper and lower joints are spherical and universal joints, respectively. This 3D model is viewed in the Mechanics Explorer that allows one to investigate the performance of the simulation model visually.

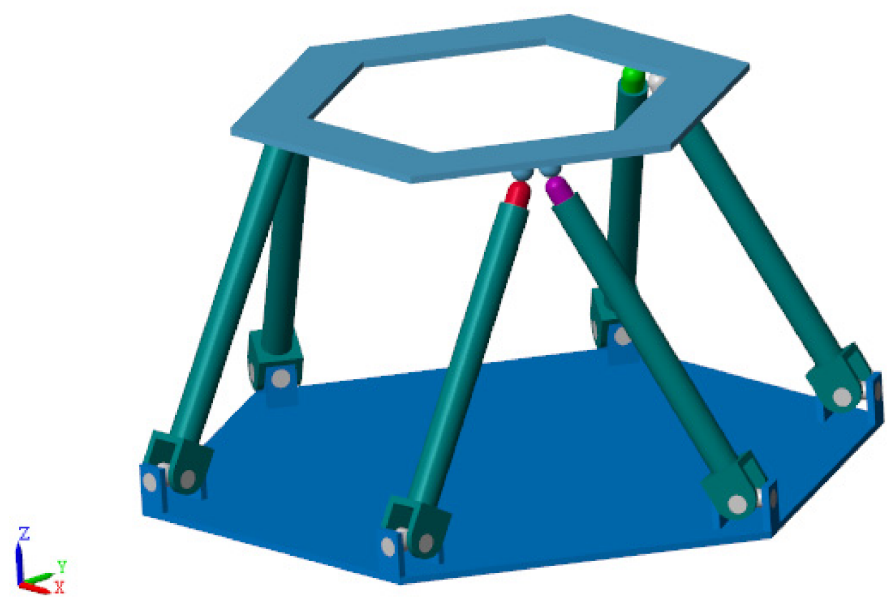

Figure 4. Multibody simulation model of the Stewart platform.

Each of the six linear actuators consists of a linear actuator body that is attached by a universal joint to the base and a piston rod that is attached to the moving plate by a spherical joint. Both the linear actuator body and the piston rod have reference frames attached to them. The linear actuator body has fixed coordinate frame $\mathbf{O}_{\mathbf{c}} x_{c} y_{c} z_{c}$ and the piston rod has fixed coordinate frame $\mathbf{O}_{\mathbf{r}} x_{r} y_{r} z_{r}$ as is shown in Figure 5. As mentioned in Section 2, these two bodies are connected by a cylindrical joint and therefore the piston rod reference frame translates with respect to the linear actuator body reference frame along the $z_{c}$ axis. As described in Figure 5, the distance between the mentioned reference frames is $y_{k}$ and is equivalent to the piston rod extension. The distance between the lower joint position $\mathbf{B}_{\mathbf{k}} \mathbf{b}$ and the origin of the linear actuator body $\mathbf{O}_{\mathbf{c}}$ is equal to the fixed length of the linear actuator body $h_{k}$. A cylindrical joint block can be actuated and can have specified internal mechanics such as equilibrium position, spring stiffness, and a damping coefficient. Spring properties have not been used in our case and therefore this parameter is set to null.

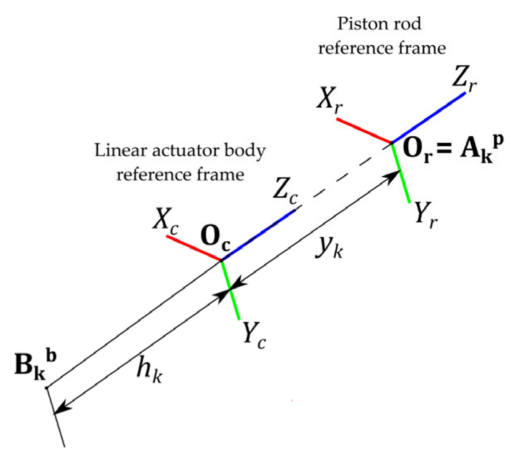

Figure 5. Detail on the linear actuator reference frames.

The cylindrical joint operates in the forward dynamics mode, where the input is the acting force, and the resulting position of the piston rod is automatically computed. In this simulation model, the dynamics of the single cylindrical joint $k$ is described by the 
mass-damper model (6), defined by a damping coefficient $b$ and a mass $m$, which are the same for each linear actuator. The acting force $F_{a k}(t)$ is provided by the linear actuator and it is calculated in the feedback controller. The loading force $F_{l k}(t)$ is acting on the linear actuator and varies with time as well. Its magnitude depends on the static configuration of the moving plate, as well as its dynamic movement and inertial properties.

$$
m \ddot{y}_{k}+b \dot{y}_{k}=F_{a k}(t)-F_{l k}(t)
$$

The MiL scenario allows us to explore and verify the control strategies, the dynamic performance, and the workspace of the Stewart platform. It can also be used for SiL, PiL, and HiL simulations, which have already been mentioned before. However, in this article we present only MiL and HiL techniques.

The multibody simulation model of the Stewart platform consists of a plant, a feedback controller, and an inverse kinematics block as shown in Figure 6. The plant block in the Stewart platform simulation model has been mostly described in the previous text. The inputs $u$ of this block are six action values that represent the force that will be applied by each linear actuator. The six outputs $y$ carry information about the actual piston rod extension. These signals are used to control the position and orientation of the Stewart platform. Inside the plant block there is also an embedded block for a measurement of the position and orientation of the moving plate with respect to the world coordination system.

The inverse kinematics block shown in Figure 6 calculates the desired piston rod extensions $\left[w_{1}, w_{2}, w_{3}, w_{4}, w_{5}, w_{6}\right]^{T}$ based on the input $\mathbf{q}_{\mathbf{w}}$. The next block is the feedback controller that consists of six proportional feedback controllers. The feedback control loop for a single linear actuator is shown in Figure 7. It consists of a proportional feedback controller and the single linear actuator plant. For the single linear actuator, the desired value $w_{k}$ is calculated by the inverse kinematics block. The error value is given by $e_{k}=w_{k}$ $-y_{k}$, where $y_{k}$ is the actual piston rod extension. The action value $u_{k}$ is equal to the acting force of the linear actuator $F_{a k}$. It should be noted that $w_{k}$ varies in time if the moving plate is about to follow the desired trajectory. Gain $K_{F}$ serves for a unit conversion of the action value $u_{k}$ given in volts $(\mathrm{V})$ to the acting force $F_{a k}$ given in newtons $(\mathrm{N})$.

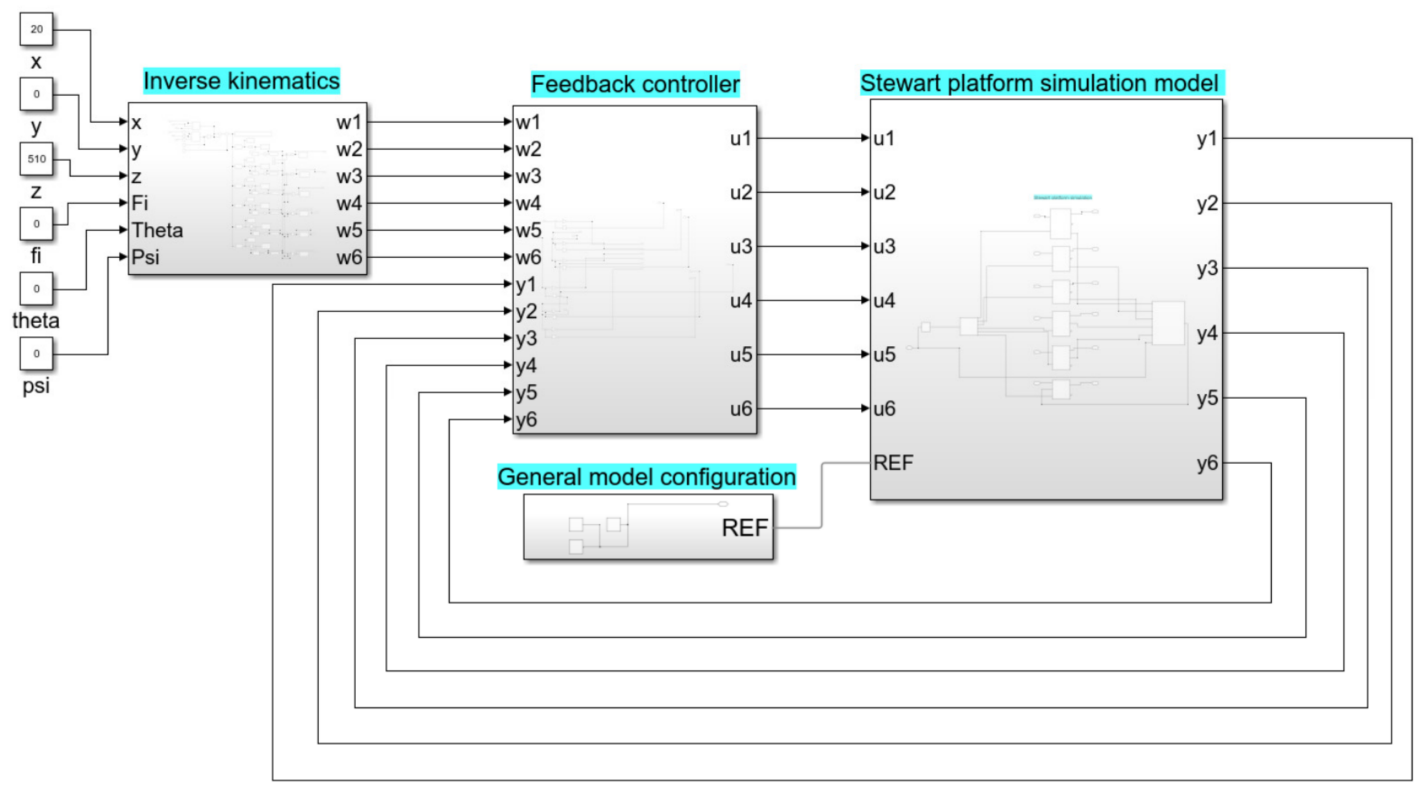

Figure 6. Simulink block diagram of the Stewart platform for MiL simulation. 


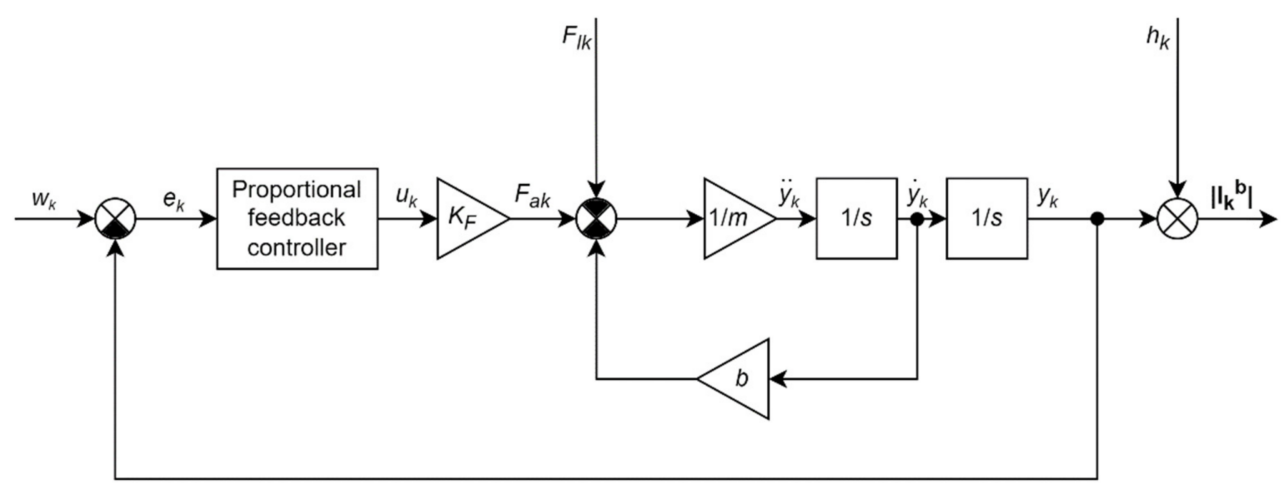

Figure 7. Feedback control loop for a single linear actuator.

Since our main focus during this experiment was not the best possible control performance, but rather an investigation of the model-based design approach, we designed a simple proportional feedback controller. For our system, it results in lower quality but functional control of the moving plate.

In the simulations, authors used the Runge-Kutta (ode4) solver with the fixed-step size $2 \mathrm{~ms}$. In Figure 8 can be seen transient responses of the moving plate for the step changes of the position of the Stewart platform. The initial desired position and orientation of the Stewart platform is $\mathbf{q}_{\mathbf{w} 0}=\left[0,0,0.49,0^{\circ}, 0^{\circ}, 0^{\circ}\right]^{\mathrm{T}}$. The second desired position and orientation of the moving plate is $\mathbf{q}_{\mathbf{w} 1}=\left[0.02,0,0.52,0^{\circ}, 0^{\circ}, 0^{\circ}\right]^{\mathrm{T}}$. From Figure 8 can be seen that the moving plate moves first from $\mathbf{q}_{\mathbf{w} 0}$ to $\mathbf{q}_{\mathbf{w} 1}$ and then back to the $\mathbf{q}_{\mathbf{w} 0}$. There is a visible deviation from the desired position and orientation of the moving plate. It must be noted that the desired angles $\varphi_{w}, \theta_{w}, \Psi_{w}$, which describe the orientation of the moving plate, are equal to $0^{\circ}$. As can be seen in Figure 8, the deviation between desired and actual angles $\varphi_{y}, \Psi_{y}$ is negligible. The deviation between desired and actual angle $\theta_{y}$ is more prominent.
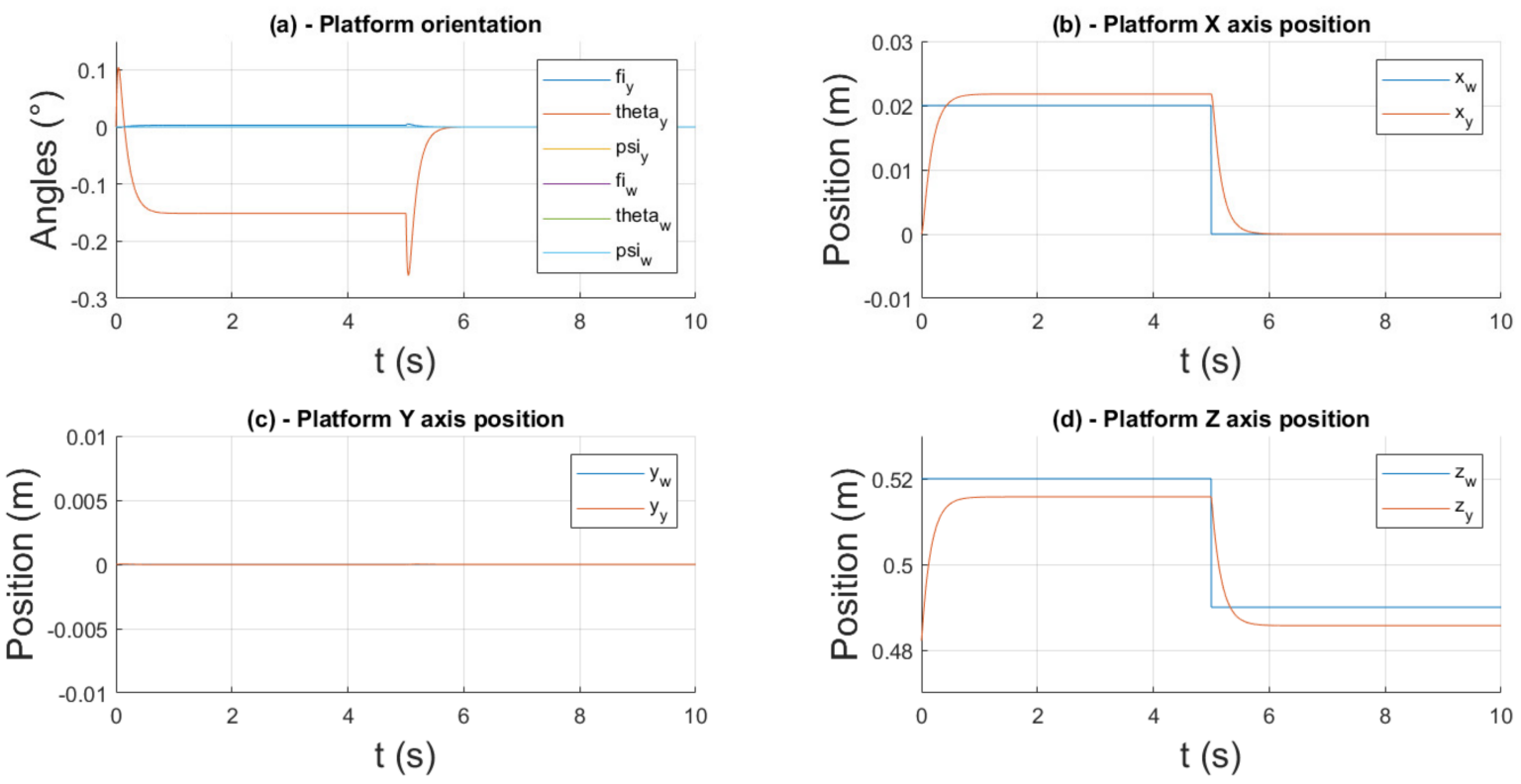

Figure 8. Moving plate position and orientation (MiL). (a) Desired $\left(\varphi_{\mathrm{w}}, \theta_{\mathrm{W}}, \Psi_{\mathrm{w}}\right)$ and actual $\left(\varphi_{\mathrm{y}}, \theta_{\mathrm{y}}, \Psi_{\mathrm{y}}\right)$ orientation of the moving plate; $(\mathbf{b})$ Desired $\left(\mathrm{x}_{\mathrm{w}}\right)$ and actual $\left(\mathrm{x}_{\mathrm{y}}\right)$ position of the moving plate in the $X_{b}$ axis; (c) Desired $\left(y_{w}\right)$ and actual $\left(y_{y}\right)$ position of the moving plate in the $Y_{b}$ axis; (d) Desired $\left(\mathrm{z}_{\mathrm{w}}\right)$ and actual $\left(\mathrm{z}_{\mathrm{y}}\right)$ position of the moving plate in the $\mathrm{Z}_{\mathrm{b}}$ axis.

In Figure 9, six transient responses of the piston rod extensions can be seen. The response times are sufficient, but due to the choice of the proportional controller there is a 
permanent control deviation that resulted in the error in position and orientation of the moving plate, as we can see in Figure 8.
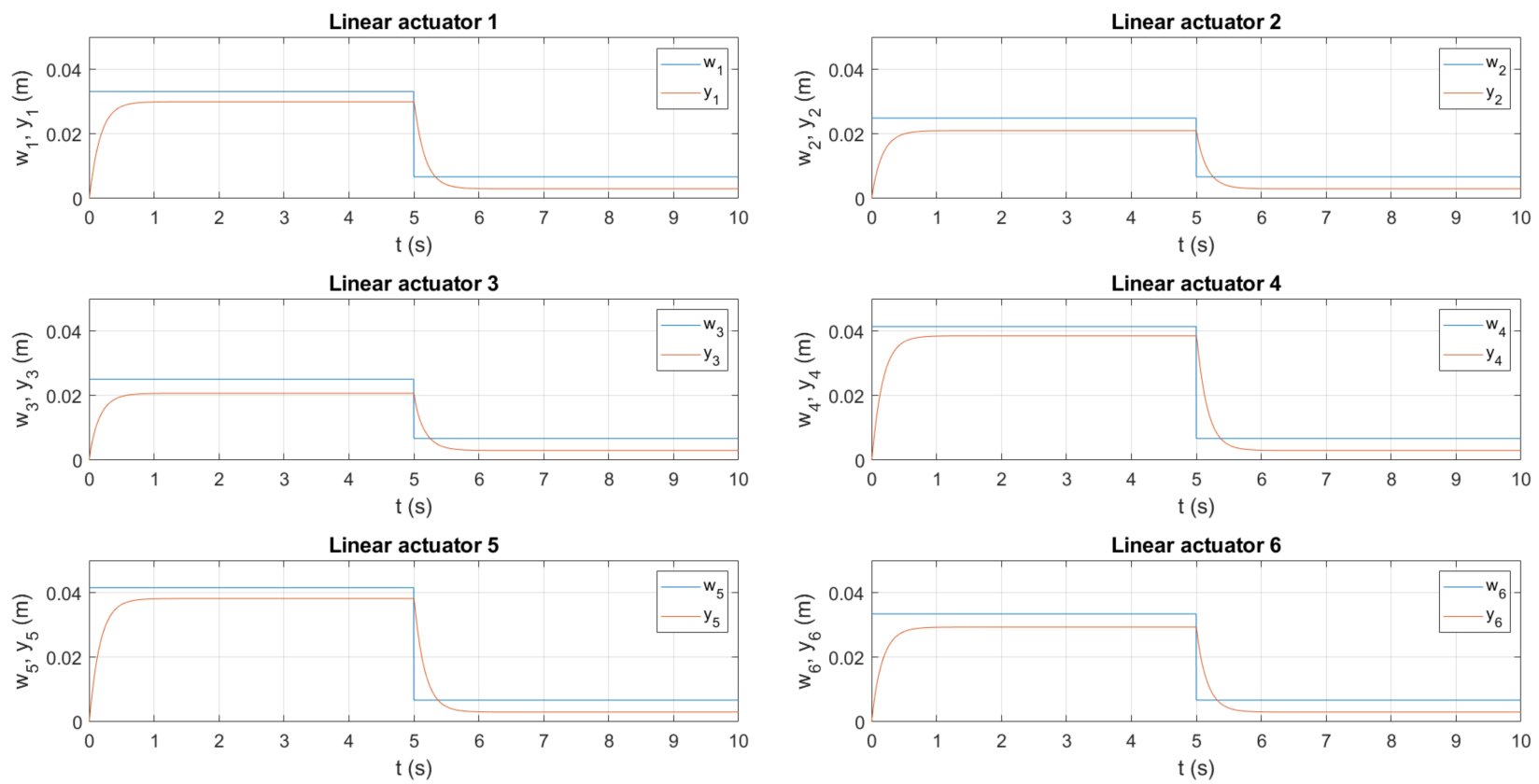

Figure 9. Actual and desired piston rod extensions of the Stewart platform linear actuators (MiL).

In Figure 10, the acting forces $F_{a}$ that are equivalent to the action input $u$, can be seen over time. It is visible that when the desired value changes the piston sharply accelerates and then decelerates until it reaches the steady state. Since bodies in the multibody simulation are subjected to gravitational forces, the steady state force of the linear actuators is non-null. It should also be noted that the applied force reaches both positive and negative values, which means that linear actuators are subjected to compressive and tensile loads.

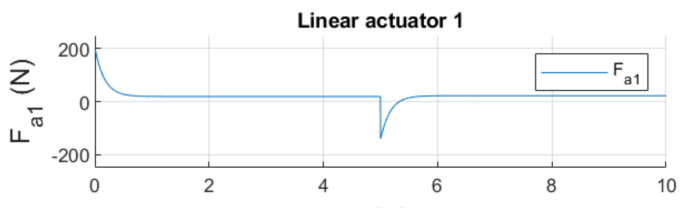

$\mathrm{t}(\mathrm{s})$

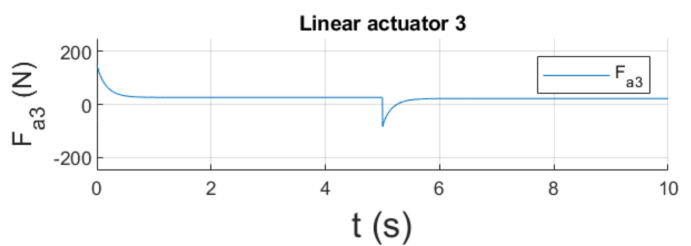

Linear actuator 5

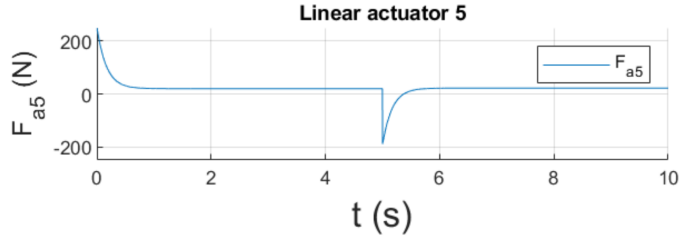

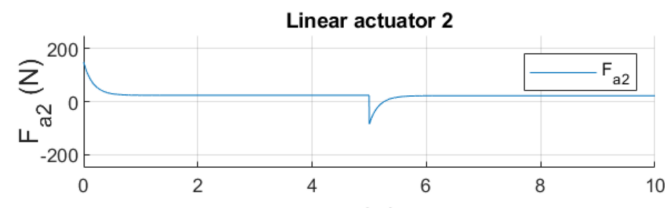

$\mathrm{t}(\mathrm{s})$
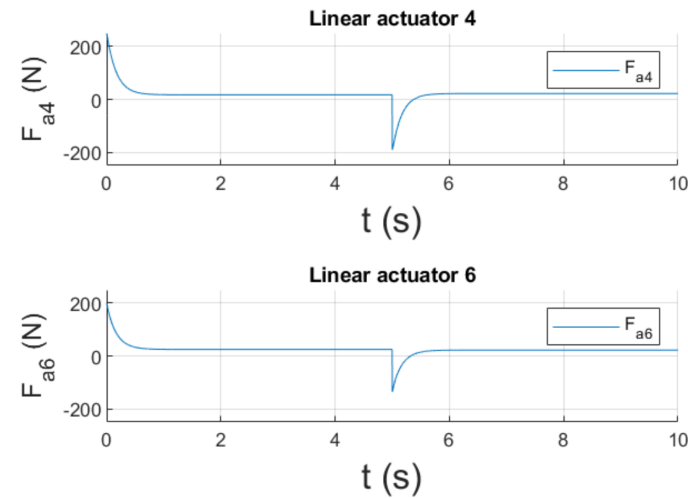

Figure 10. Acting forces of the Stewart platform linear actuators (MiL).

In this section, we have described the simulation model of the Stewart platform and the MiL scenario. Section 4 describes the Hardware-in-the-Loop experiment and compare its results to the results obtained in the MiL scenario. 


\section{Hardware-in-the-Loop (HiL) Simulation of the Stewart Platform}

In this section, we present the HiL technique in the Stewart platform development procedure according to the model-based design approach. In this scenario, we can test and potentially validate the PLCnext controller for the Stewart platform control. In this case, the plant is generated as a code and uploaded to the real-time target dSPACE MicroLabBox (MLB). The inverse kinematics part of the control algorithm is generated as a code from the Matlab/Simulink environment as well and uploaded to the PLCnext as a subroutine. PLCnext consists of both generated Matlab/Simulink code and code programmed in its own programming environment PLCnext Engineer. PLCnext also allows running HMI for control and visualisation of the process. Since PLCnext has web server functionality, the HMI can be accessed through the web browser.

In Figure 11 a workplace for the HiL simulation is shown. It consists of the PLCnext, the MLB, a PC, and a laptop. PLCnext and MLB are connected to each other through the $\mathrm{I} / \mathrm{O}$ interface that consists of analog inputs and outputs operating in the range $0-10 \mathrm{~V}$. PLCnext is also connected to the PC through Ethernet. This connection serves to upload the code to the controller and access the HMI, where an operator enters the desired position and orientation of the moving plate $\mathbf{q}_{\mathbf{w}}$. The MLB is also connected to the laptop via Ethernet. In addition to uploading the code to the real-time target, it also serves for visualising and data acquisition in the ControlDesk environment. The mentioned environment also allows the user to manipulate the parameters of the plant. The plant parameters have not been changed during this simulation experiment.

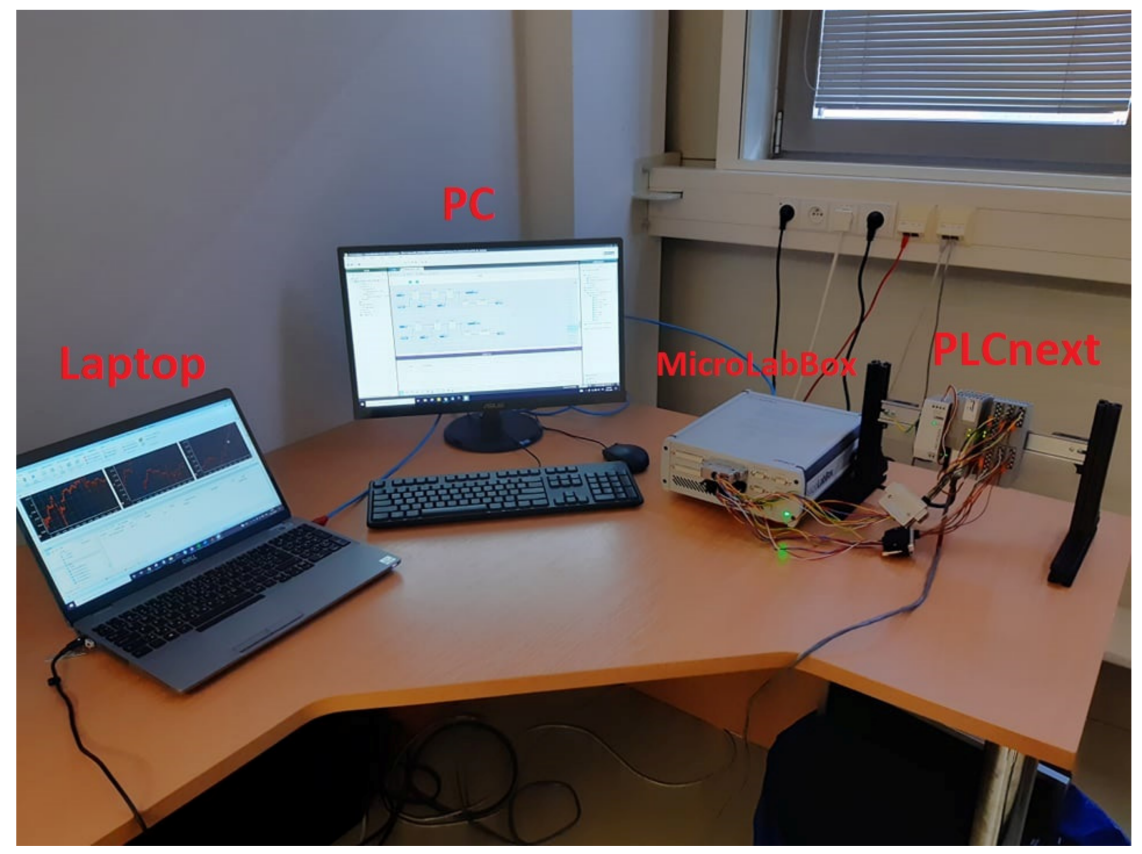

Figure 11. HiL simulation workplace.

The HiL simulation scheme is also described by the diagram in Figure 12. Where $\mathbf{q}_{\mathbf{w}}$ is a vector carrying information about the desired position and orientation of the moving plate. The operator enters $\mathbf{q}_{\mathbf{w}}$ values via the HMI interface on the PC. The controller PLCnext contains inverse kinematics and proportional feedback controller code and is based on the operator input and actual leg extension $y_{1}, \ldots, y_{6}(t)$ calculates action values $u_{1}, \ldots, u_{6}(t)$ for each linear actuator, which are part of the simulated plant in the real-time target MicroLabBox. MLB is connected with the laptop. As mentioned in the previous paragraph, the ControlDesk environment, which is running on the laptop, can be used for the data acquisition and the visualisation of the action values, actual piston rod extensions and an actual position and orientation of the moving plate $\mathbf{q y}$. 


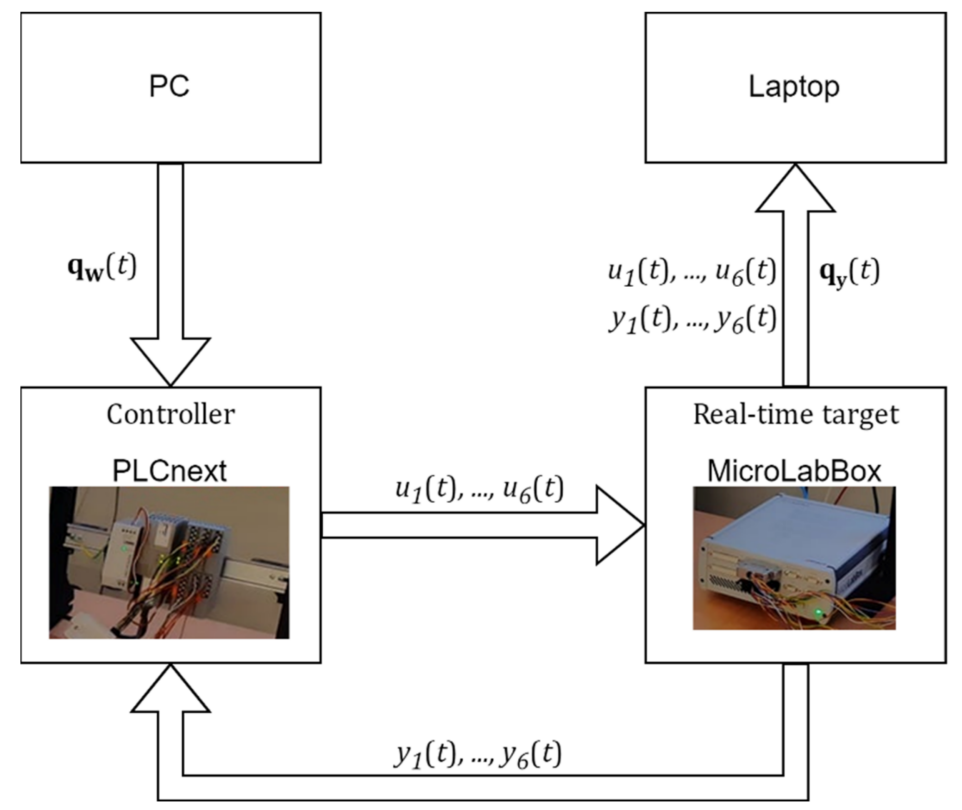

Figure 12. Stewart platform HiL simulation scheme.

Code generation allows us to seamlessly transit from the design phase to the system integration phase. This approach saves a significant amount of time that would otherwise be required if both real-time target and controller had to be coded manually. The main reason is that manual coding is prone to human errors and is also very technically demanding, especially in the case of the plant code. Moreover, PLC programming languages typically do not support tools for linear algebra (i.e., matrix operations), which would make implementing inverse kinematics challenging as well. With the described tools, we can test and validate the chosen controller in this early phase of development before the first prototype has been built, which can significantly reduce time and costs. Nevertheless, we must be sure that the fidelity of the simulated plant is accurate enough before we draw any important conclusions.

In Figure 13 the inverse kinematics algorithm implemented in the Simulink environment can be seen. The implementation of the inverse kinematics is based on equations presented in Section 2; however, they are adjusted for the Simulink implementation. The terms of the vector $\mathbf{q}_{\mathbf{w}}$ are defined as inputs, and the calculated outputs $w_{1}, \ldots, w_{6}$ are the desired piston rod extensions. The block actuator body size shown in Figure 13 is equal to the length of the linear actuator body $h_{k}$. The signal representing the piston rod extensions is scaled to the required range. There is also a saturation block that prevents the output signal from being of a higher magnitude than allowed. When we generate the code out of this model, we get a library block that can be used in the PLCnext code. Programming is done in PLCnext Engineer, which is a software platform for Phoenix Contact automation controllers. In this environment, we can use the generated library block and use it as a subroutine in PLCnext. We must specify the task to which it belongs to and assign the input and output variables. The inner structure of the inverse kinematics library cannot be investigated in this environment. Only its inputs and outputs are available. Generated libraries, therefore, act like a black box. 


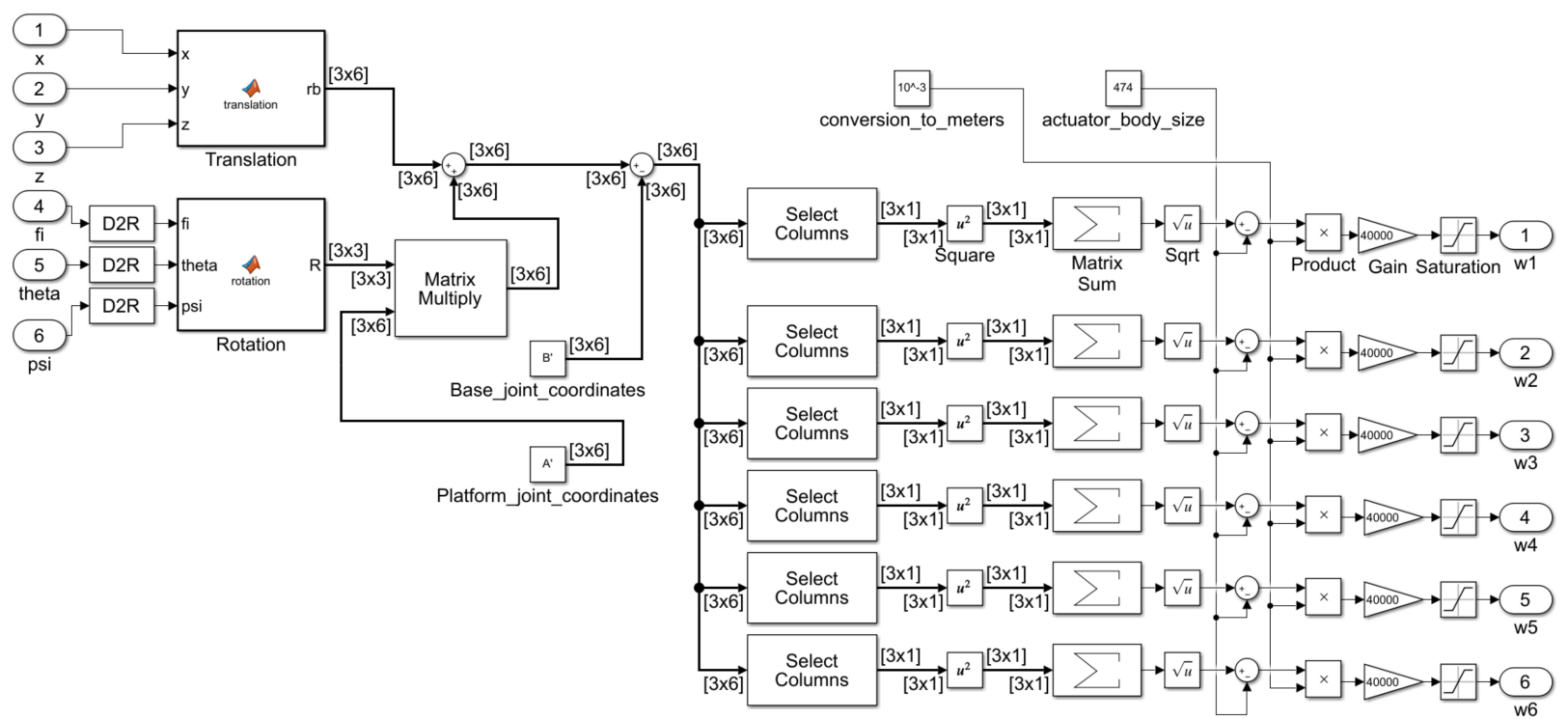

Figure 13. The inverse kinematics algorithm coded in the Matlab/Simulink environment.

In the PLCnext Engineer, we have used the generated inverse kinematics block as a single subroutine. The feedback controller part has been coded directly in the new subroutine. The inverse kinematics subroutine for calculating desired leg lengths runs in the same cycle as the feedback controller subroutine with $1 \mathrm{~ms}$ time period. The snippet of the controller code is shown in Figure 14.

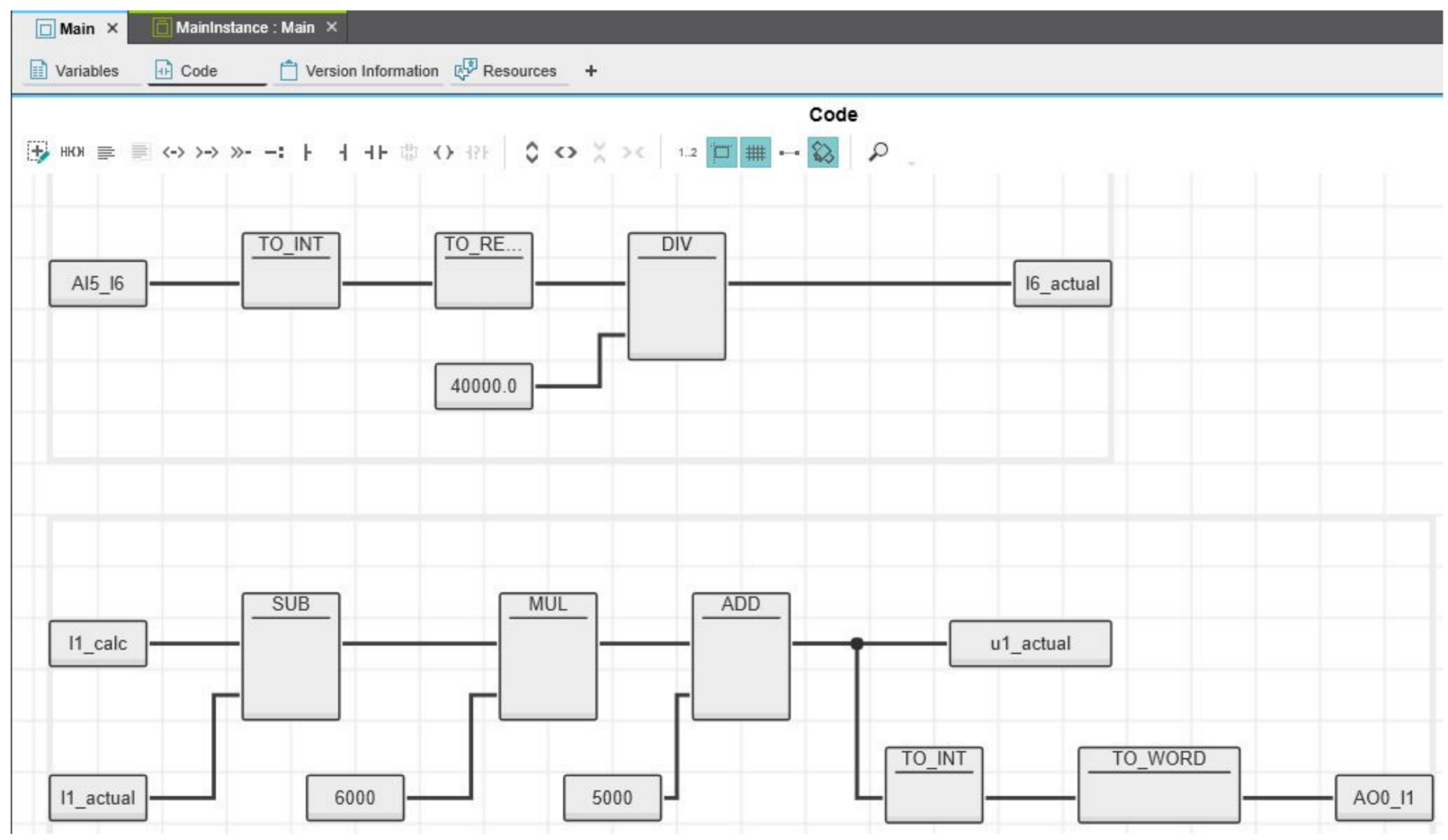

Figure 14. Part of the code of the Stewart platform controller.

In Figure 14 the signal from analog input is shown to be processed and scaled. The same goes for the output signal. In Figure 15 is a simple HMI interface that is used to define $\mathbf{q}_{\mathbf{w}}$. As mentioned before, HiL allows us to design and test graphical user interfaces and control systems higher in the control hierarchy even before the real prototype is built. In this configuration, it would also be possible to collect data from the simulation model and 
compare it to the data from the real Stewart platform, and thus this configuration could also be a significant part in the emerging research topic of the Digital twin [30-32].

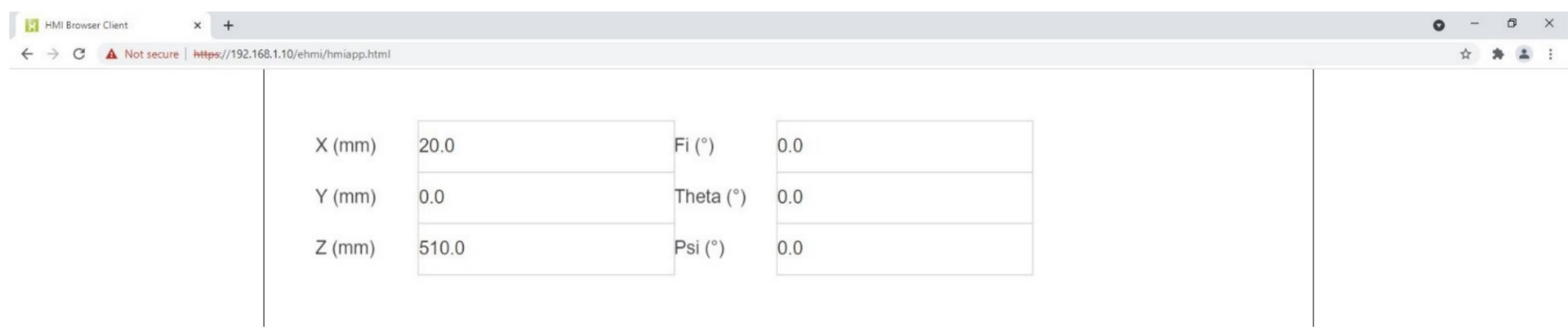

Figure 15. The HMI interface for the Stewart platform control.

In the HiL plant simulation, authors used Runge-Kutta (ode4) solver with the fixedstep size $2 \mathrm{~ms}$, as in the MiL scenario. In Figure 16 is the HiL simulation model of the Stewart platform created in the Matlab/Simulink. This simulation model is adjusted for the real-time simulation in the MLB. The Stewart platform plant is the same as in the MiL scenario, only the blocks for the inverse kinematics and the feedback control have been adjusted and moved to the PLC controller. In the HiL simulation model, there are blocks for analog inputs that receive a signal that carries information of the action values $u$. The blocks for the output signals, on the other hand, carry the information regarding the actual piston rod extension $y$. Both input and output signals need to be scaled and processed accordingly.

\section{RTI Data}

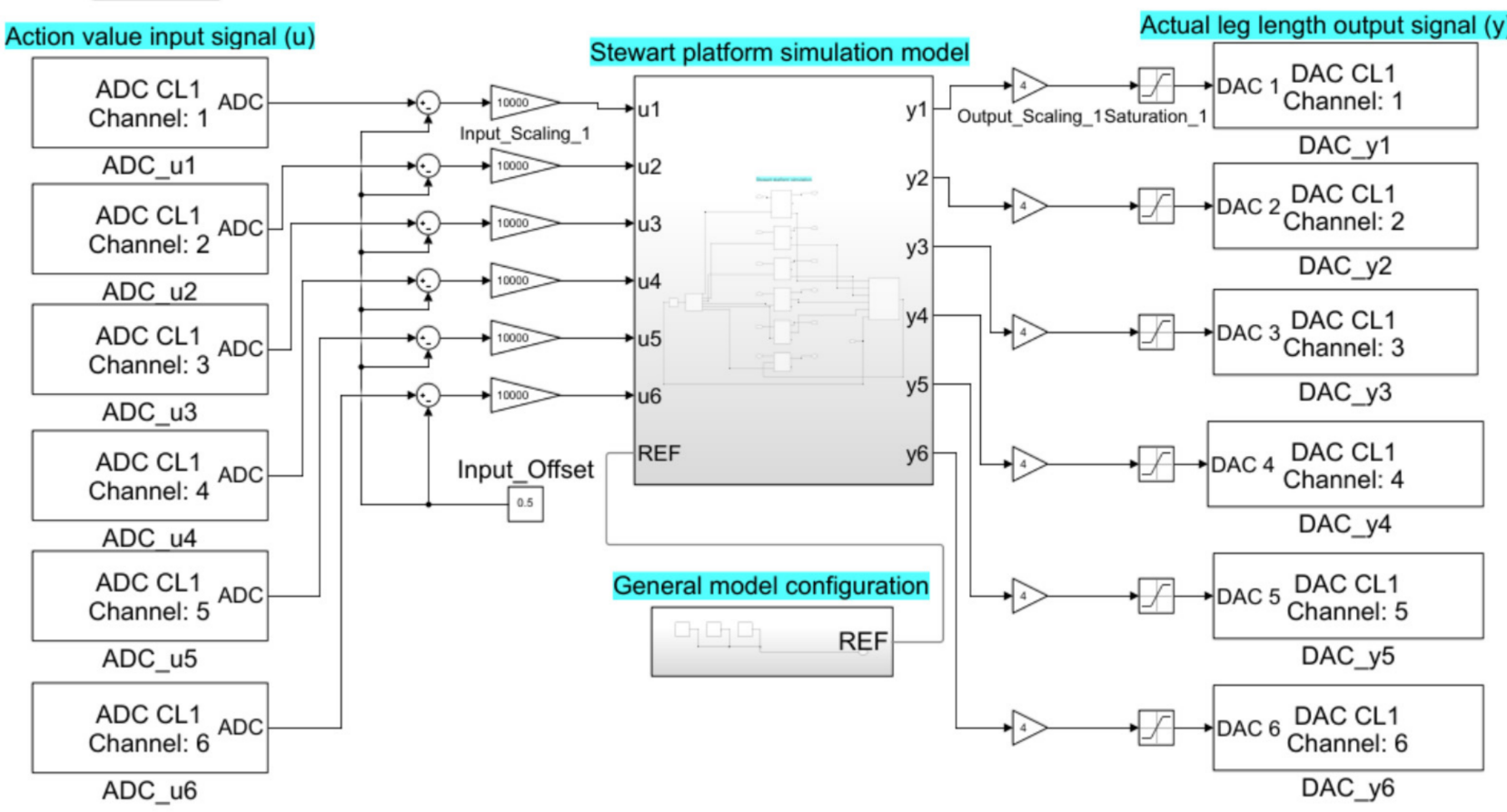

Figure 16. Simulink block diagram of the Stewart platform for the HiL simulation.

In Figure 17 the transient responses of the moving plate for the step change of the position and orientation of the Stewart platform obtained during the HiL simulation can be seen. The initial and desired position and orientation $\mathbf{q}_{\mathbf{0}}$ and $\mathbf{q}_{\mathbf{1}}$ of the Stewart platform are the same as in the MiL scenario and the same operation has been performed as well but with the different timing. Since the desired value signal is not transferred to the MicroLabBox, we cannot display it in the same graph as $\mathbf{q}_{\mathbf{y}}$ values. 


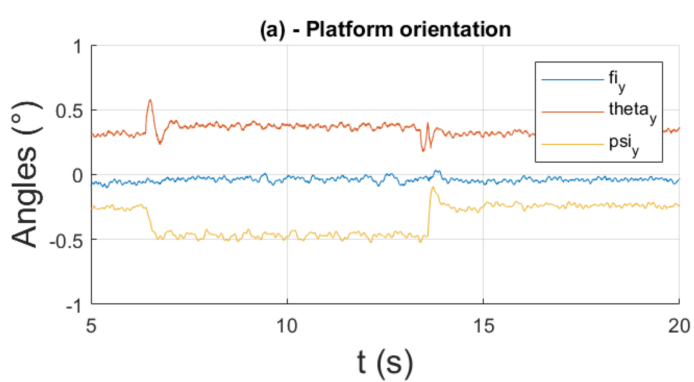

(c) - Platform $Y$ axis position

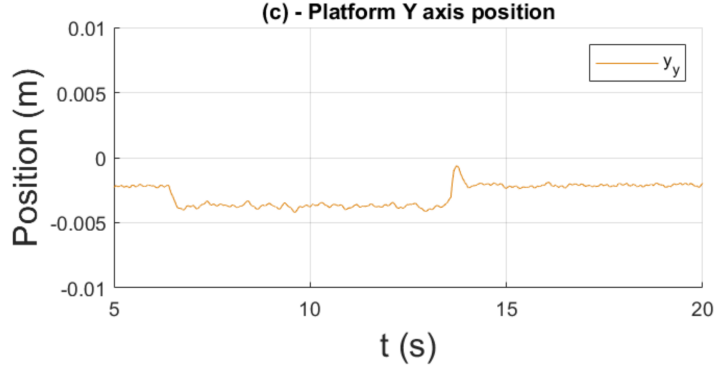

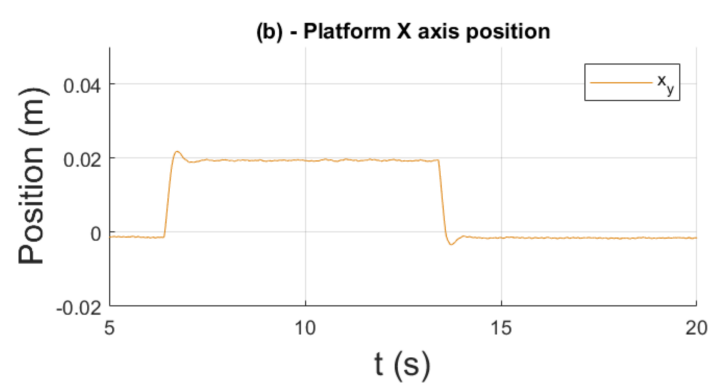

(d) - Platform Z axis position

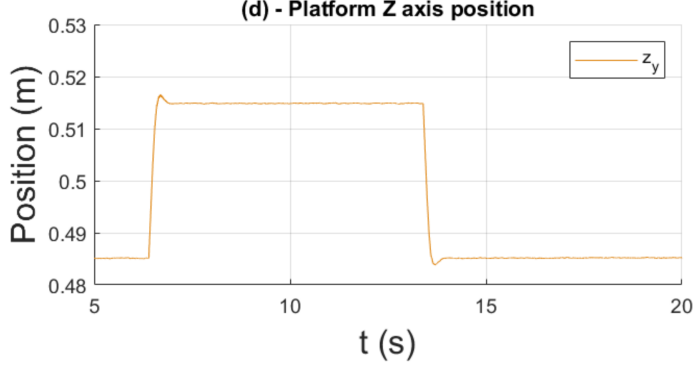

Figure 17. Moving plate position and orientation (HiL). (a) Actual $\left(\varphi_{y}, \theta_{y}, \Psi_{y}\right)$ orientation of the moving plate; $(\mathbf{b})$ Actual $\left(\mathrm{x}_{\mathrm{y}}\right)$ positon of the moving plate in the $\mathrm{X}_{\mathrm{b}}$ axis; (c) Actual $\left(\mathrm{y}_{\mathrm{y}}\right)$ position of the moving plate in the $Y_{b}$ axis; $(d)$ Actual $\left(z_{y}\right)$ position of the moving plate in the $Z_{b}$ axis.

The important conclusion from Figure 17 is that analogically to the MiL scenario the error in linear actuator control results in the similar deviations of position and orientation of the moving plate. However, it must be noted that the steady state deviation in the platform orientation is more prominent and the transient responses are slightly oscillating.

In Figure 18 are transient responses of the piston rod extensions. Graphs show that transient responses are slightly oscillating, which is the reason the actual position and orientation of the moving plate is oscillating as well. The steady state values are comparable to the MiL scenario with the exception of the first linear actuator where a small error in magnitude is visible, which must result in an error in position and orientation of the moving plate. Authors assume this discrepancy is caused by one of the issues mentioned at the end of this chapter.
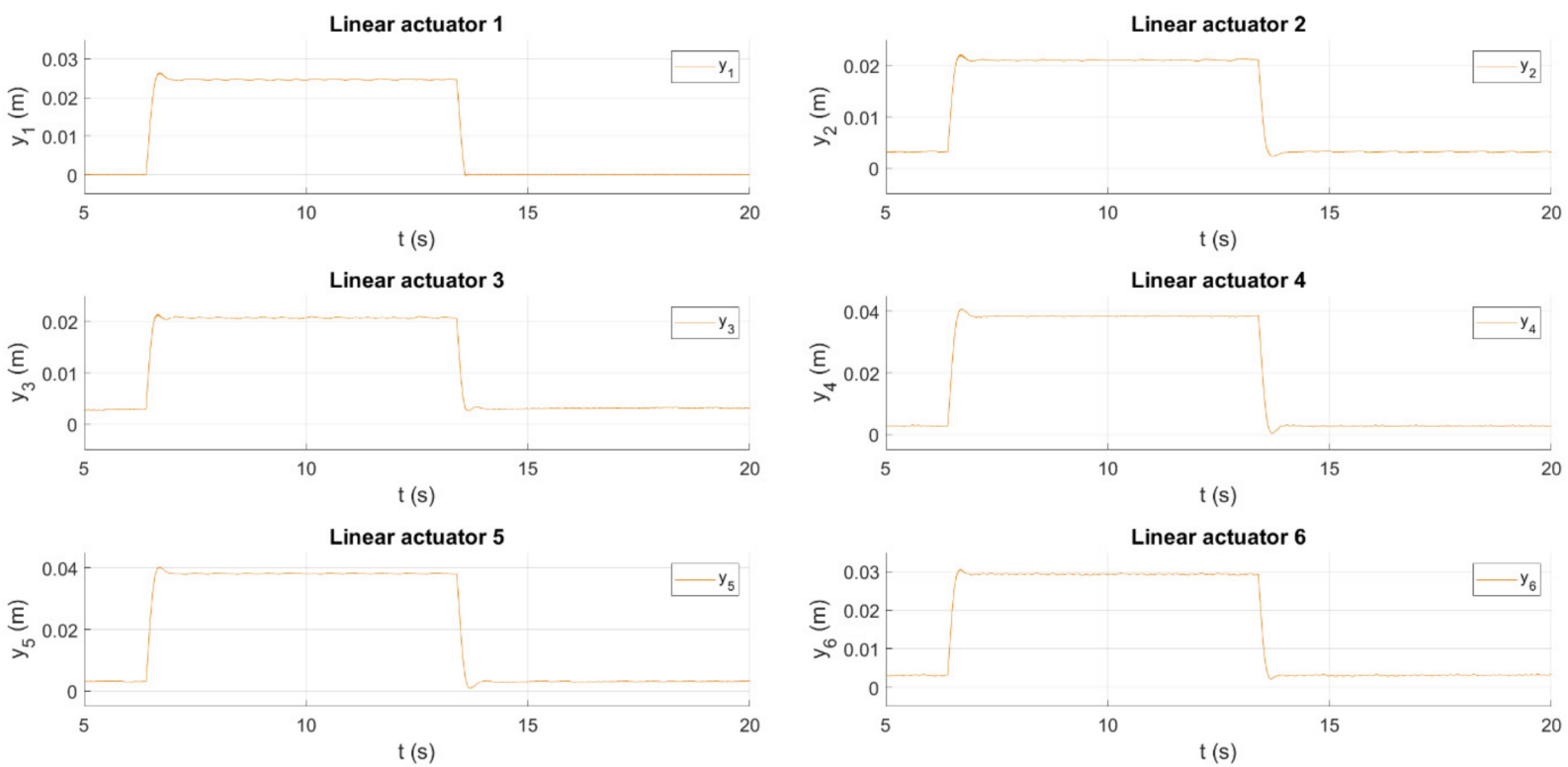

Figure 18. Actual piston rod extensions of the Stewart platform linear actuators (HiL). 
In Figure 19 the acting forces $F_{a}$ of the linear actuators, that are equivalent to the action value inputs $u$, are shown. It can be seen that the acting forces slightly oscillate and that the peak values and steady states are similar to the MiL simulation results.
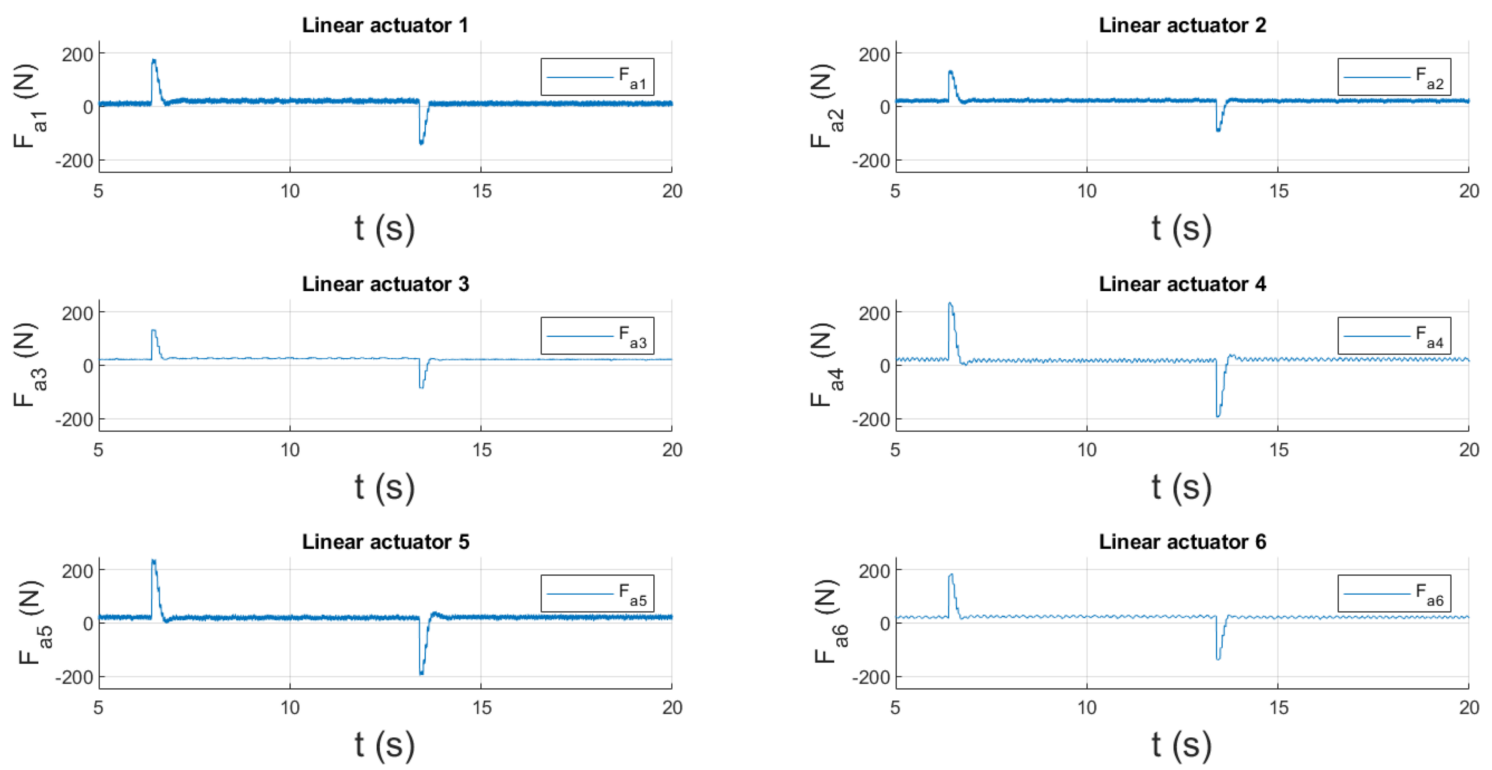

Figure 19. Acting forces of the Stewart platform linear actuators (HiL).

Even though the obtained data cannot be directly compared to the MiL scenario, from the graphs it is obvious that the dynamics of the system is very similar. The main discrepancy in the MiL and HiL scenario is in the oscillation of the transient responses and the error in the magnitude of the first piston rod extension. These discrepancies can be caused by multiple issues such as different sampling periods of the controller and the simulated plant, connection of the real controller and the associated conversion of quantities from numerical format to voltage signal, quantization of signals during A/D and D/A conversions, rounding errors and also slight time delay. However, since most of the mentioned issues also occur during the control of the real system, the obtained results might represent the real control conditions. From this perspective, it is important that the control shows stability, required dynamics and accuracy which also depends on the type and parameters of the used controller.

The HiL technique allowed us to get acquainted with properties and behaviour of the Stewart platform in real-time scenario with an actual real controller design. It also allowed us to experiment with the system in the virtual environment and therefore we avoided the potential risk of destroying or damaging the real equipment. This scenario also possibly allows us to investigate the synchronization of the individual linear actuators which must be precise in high-precision Stewart platform applications.

\section{Conclusions}

In this paper we used methods for the mechatronics system development as described in the V-Cycle. We applied the model-based techniques Model-in-the-Loop and Hardwarein-the-Loop simulations in the case of the Stewart platform control strategy development. We discovered that the MiL method allowed us to get acquainted with the behaviour and properties of the system in the early stage of the design and we could also verify the controller part which was inverse kinematics and feedback control design.

In the next phase, we were able to translate smoothly from the design phase to the system integration phase, due to the fact that we were able to generate the inverse kinematics part of the designed controller code and used it as a subroutine in the real controller PLCnext. The feedback controller and HMI functions have then been coded manually and implemented in the single PLCnext programme with the inverse kinematic 
part. Since inverse kinematic calculations require matrix operations, which are usually not supported by any libraries in the PLCs, the code generation tools really accelerated this transition, and therefore we avoided coding the inverse kinematics in the standard PLC languages that would be error prone and cumbersome.

After obtaining the results from the HiL we found out that it slightly differs from the MiL scenario results. The main deviation was present in the slight oscillation of the transient response in the HiL results and the error in the magnitude in the piston rod extension of the first linear actuator. These discrepancies can be caused by multiple issues, mentioned in the article. From the perspective of the HiL simulation, it is important that the control shows stability, a required dynamics and accuracy, which also depend on the type and parameters of the used controller. The authors also must note, that during some of the scenarios of the HiL simulation experiments they ran into the numerical stability issues caused by the inability of the MicroLabBox to perform the calculations in the fixed step time. This issue did not occur in the MiL scenario. The authors therefore recommend that the readers use a more powerful real-time target than a MicroLabBox.

During the experiments, we discovered that the model-based approach is a strong technique, because it allowed us to explore the behaviour and properties of the system before the first prototype has been made in a virtual environment and therefore, we discovered potential issues early in the development phase that would result in reduced costs and time. That includes avoiding the potential for damaging and destroying the real equipment. However, it is fair to say that the employment of this approach is timeconsuming in the initial stages, because development of the plant simulation model is demanding as well, and it is hard to verify the fidelity of the simulation model if the real prototype is nonexistent. Therefore, in the early design stages, it is required to approach the simulation results as critically as possible and it is necessary to continually improve precision of the used simulation model. Otherwise, false conclusions could be drawn, which would result in negative consequences in a later development phase.

In the HiL scenario, we are also able to test the developed functions of the controller and the higher control hierarchy as well. The obtained data can be processed and higherlevel data processing algorithms can make decisions and conclusions based on them. Moreover, in case the developed simulation model of the plant accompanied the real product during the development cycle and has been previously verified and validated, it could possibly be used even after the development with the real product. The developed simulation model could be run in parallel with the real product as its digital twin. This could possibly add another value for the existing product and therefore it would return resources spent on the high-fidelity simulation model. In case of the Stewart platform, it could be used for the workspace investigation, trajectory planning, predictive maintenance, etc.

Author Contributions: Data curation, D.W.; Formal analysis, P.N.; Supervision, P.N.; Writingoriginal draft, D.W.; Writing-review and editing, P.N. All authors have read and agreed to the published version of the manuscript.

Funding: This work was funded by the European Regional Development Fund in the Research Centre of Advanced Mechatronic Systems project, CZ.02.1.01/0.0/0.0/16_019/0000867 within the Operational Programme Research, Development and Education and the project SP2022/60 Applied Research in the Area of Machines and Process Control supported by the Ministry of Education, Youth and Sports.

Data Availability Statement: The presented data can be provided by the authors on request.

Acknowledgments: The authors would also like to acknowledge PHOENIX CONTACT, s.r.o. situated in Brno, Czech Republic for borrowing the PLCnext controller for the experiment.

Conflicts of Interest: The authors declare no conflict of interest.

\section{References}

1. Verein Deutscher Ingenieure. VDI 2206: Design Methodology for Mechatronic Systems; Beuth Verlag: Berlin, Germany, 2004. 
2. Li, H.; Tian, L.; Vogel-Heuser, B. Automatic Synchronization of Mechanical CAD Models and a SysML-based Mechatronic Model using AutomationML. In Proceedings of the 2019 IEEE International Conference on Systems, Man and Cybernetics (SMC), Bari, Italy, 6-9 October 2019; pp. 3343-3350. [CrossRef]

3. Watkins, C.B.; Varghese, J.; Knight, M.; Petteys, B.; Ross, J. System Architecture Modeling for Electronic Systems Using MathWorks System Composer and Simulink. In Proceedings of the 2020 AIAA/IEEE 39th Digital Avionics Systems Conference (DASC), San Antonio, TX, USA, 11-15 October 2020; pp. 1-10. [CrossRef]

4. Abourida, S.; Dufour, C.; Bélanger, J. Real-Time and Hardware-In-The-Loop Simulation of Electric Drives and Power Electronics: Process, problems and solutions. In Proceedings of the 2005 International Power Electronics Conference, Niigata, Japan, 4-8 April 2015.

5. Fotso, A.B.; Rettberg, A. State of the art for mechatronic design concepts. In Proceedings of the 2012 IEEE/ASME 8th IEEE/ASME International Conference on Mechatronic and Embedded Systems and Applications, Suzhou, China, 8-10 July 2012; pp. 232-240. [CrossRef]

6. Michaels, L.; Pagerit, S.; Rousseau, A.; Sharer, P.; Halbach, S.; Vijayagopal, R.; Kropinski, M.; Matthews, G.; Kao, M.; Matthews, O.; et al. Model-Based Systems Engineering and Control System Development via Virtual Hardware-in-the-Loop Simulation. 2010. Available online: https:/ / saemobilus.sae.org/content/2010-01-2325/ (accessed on 20 February 2022). [CrossRef]

7. Jian, B.; Wang, H. Hardware-in-the-loop real-time validation of fuel cell electric vehicle power system based on multi-stack fuel cell construction. J. Clean. Prod. 2022, 331, 129807. [CrossRef]

8. Sankaranarayanan, V.; Shirazi, M.; Gao, Y.; Ghosh, A.; Erickson, R.W.; Maksimovic, D. Controller Hardware-in-the-Loop Validation of a Modular Control Architecture for a Composite DC-DC Converter. In Proceedings of the 2019 20th Workshop on Control and Modeling for Power Electronics (COMPEL), Toronto, ON, Canada, 16-19 June 2019; pp. 1-7. [CrossRef]

9. Vidanapathirana, A.; Dewasurendra, S.D.; Abeyaratne, S.G. Model in the loop testing of complex reactive systems. In Proceedings of the 2013 IEEE 8th International Conference on Industrial and Information Systems, Kandy, Sri Lanka, 17-20 December 2013; pp. 30-35. [CrossRef]

10. Taut, M.A.; Chindris, G.; Taut, A.C. Software-in-the-Loop System for Motor Control Algorithms. In Proceedings of the 2019 IEEE 25th International Symposium for Design and Technology in Electronic Packaging (SIITME), Cluj-Napoca, Romania, 23-26 October 2019; pp. 419-426. [CrossRef]

11. Jiang, Z.; Leonard, R.; Dougal, R.; Figueroa, H.; Monti, A. Processor-in-the-loop simulation, real-time hardware-in-the-loop testing, and hardware validation of a digitally-controlled, fuel-cell powered battery-charging station. In Proceedings of the 2004 IEEE 35th Annual Power Electronics Specialists Conference (IEEE Cat. No.04CH37551), Aachen, Germany, 20-25 June 2004; Volume 3, pp. 2251-2257. [CrossRef]

12. Choe, J.-M.; Arnedo, L.; Lee, Y.; Sorchini, Z.; Mignogna, A.; Agirman, I.; Kim, H. Model-Based Design and DSP Code Generation using Simulink ${ }^{\circledR}$ for Power Electronics Applications. In Proceedings of the 2019 10th International Conference on Power Electronics and ECCE Asia (ICPE 2019_ECCE Asia), Busan, Korea, 27-31 May 2019; pp. 923-926. [CrossRef]

13. Li, H.; Li, W.; Cui, D. Automatic code generation of SPWM for single phase inverter by model-based design. In Proceedings of the 2013 2nd International Conference on Measurement, Information and Control, Harbin, China, 16-18 August 2013; pp. 1035-1038. [CrossRef]

14. Phoenix Contact. Controller-AXC F 2152-2404267, Germany. 2021. Available online: https://asset.conrad.com/media10/add/16 0267/c1/-/gl/002239019DS01/karta-katalogowa-2239019-kontroler-plc-phoenix-contact-axc-f-2152-2404267.pdf (accessed on 20 February 2022).

15. Stewart, D. A platform with six degrees of freedom. Proc. Inst. Mech. Eng. 1965, 180, 371-386. [CrossRef]

16. Ding, B.; Cazzolato, B.; Stanley, R.; Grainger, S.; Costi, J. Stiffness Analysis and Control of a Stewart Platform-Based Manipulator with Decoupled Sensor-Actuator Locations for Ultrahigh Accuracy Positioning Under Large External Loads. J. Dyn. Syst. Meas. Control 2014. [CrossRef]

17. Iqbal, S.; Bhatti, A.; Ahmed, Q. Dynamic Analysis and Robust Control Design for Stewart Platform with Moving Payloads. IFAC Proc. Vol. 2008, 41, 5324-5329. [CrossRef]

18. Shariatee, M.; Akbarzadeh, A. Optimum Dynamic Design of a Stewart Platform with Symmetric Weight Compensation System. J Intell. Robot. Syst. Theory Appl. 2021, 103, 66. [CrossRef]

19. Lu, Z.-Q.; Wu, D.; Ding, H.; Chen, L.-Q. Vibration isolation and energy harvesting integrated in a Stewart platform with high static and low dynamic stiffness. Appl. Math. Model. 2020, 89, 249-267. [CrossRef]

20. Markou, A.; Elmas, S.; Filz, G.H. Revisiting Stewart-Gough platform applications: A kinematic pavilion. Eng. Struct. 2021, 249, 113304. [CrossRef]

21. Hajdu, S.; Bodnár, D.; József, M.; Békési, Z. Kinematical simulation methods for Stewart Platform in medical equipments. Int. Rev. Appl. Sci. Eng. 2017, 8, 135-140. [CrossRef]

22. Noskievic, P.; Walica, D. Investigation of the Stewart Platform Workspace Using MATLAB-Simulink and Simscape Multibody Library. In Proceedings of the 2020 21st International Conference on Research and Education in Mechatronics (REM), Cracow, Poland, 9-11 December 2020; pp. 1-5. [CrossRef]

23. Li, B.; Cao, Y.; Zhang, Q.; Wang, C. Singularity Representation and Workspace Determination of a Special Class of the GoughStewart Platforms. Int. J. Adv. Robot. Syst. 2013, 10, 1. [CrossRef]

24. Oftadeh, R.; Aref, M.; Taghirad, H. Explicit dynamics formulation of Stewart-Gough platform: A Newton-Euler approach. In Proceedings of the IEEE/RSJ 2010 International Conference on Intelligent Robots and Systems, IROS 2010—Conference Proceedings, Taipei, Taiwan, 18-22 October 2010; pp. 2772-2777. [CrossRef] 
25. Hajimirzaalian, H.; Moosavi, H.; Massah, M. Dynamics analysis and simulation of parallel robot Stewart platform. In Proceedings of the 2010 the 2nd International Conference on Computer and Automation Engineering, ICCAE 2010, Singapore, 26-28 February 2010; Volume 5, pp. 472-477. [CrossRef]

26. Wang, J.; Gosselin, C.M. A New Approach for the Dynamic Analysis of Parallel Manipulators. Multibody Syst. Dyn. 1998, 2, 317-334. [CrossRef]

27. Fadaei, M.H.K.; Pahnehkolaei, S.A.H.; Hesarlou, M.J.; Torkan, Z. Dynamics modeling of a stewart platform in Simulink MSC ADAMS. In Proceedings of the 2017 IEEE 4th International Conference on Knowledge-Based Engineering and Innovation (KBEI), Tehran, Iran, 22 December 2017; pp. 0294-0298. [CrossRef]

28. Noskievič, P.; Walica, D. Design and Realisation of the Simulation Model of the Stewart Platform using the MATLAB-Simulink and the Simscape Multibody Library. In Proceedings of the 2020 21th International Carpathian Control Conference (ICCC), High Tatras, Slovakia, 27-29 October 2020; pp. 1-5. [CrossRef]

29. Sonar, A.; Burdick, K.D.; Begin, R.; Resch, E.M.; Thompson, E.M.; Thacher, E.; Searleman, J.; Fulk, G.; Carroll, J. Development of a virtual reality-based power wheel chair simulator. J. Comput. Phys. 2005, 1, 222-229. [CrossRef]

30. Classens, K.; Heemels, W.P.M.H.M.; Oomen, T. Digital Twins in Mechatronics: From Model-based Control to Predictive Maintenance. In Proceedings of the 2021 IEEE 1st International Conference on Digital Twins and Parallel Intelligence (DTPI), Beijing, China, 15 July-15 August 2021; pp. 336-339. [CrossRef]

31. Dufour, C.; Soghomonian, Z.; Li, W. Hardware-in-the-Loop Testing of Modern On-Board Power Systems Using Digital Twins. In Proceedings of the 2018 International Symposium on Power Electronics, Electrical Drives, Automation and Motion (SPEEDAM), Amalfi, Italy, 20-22 June 2018; pp. 118-123. [CrossRef]

32. Nguyen, V.H.; Tran, Q.T.; Besanger, Y.; Jung, M.; Nguyen, T.L. Digital twin integrated power-hardware-in-the-loop for the assessment of distributed renewable energy resources. Electr. Eng. 2021. [CrossRef] 